\title{
Problem, Solution, Validation Impact Canvas
}

Colin Keogh ${ }^{1}$, Roisin Lyons ${ }^{2}$, David R. Pollard ${ }^{1}$

1 University College Dublin

2 Dublin City University

Potential competing interests: The author(s) declared that no potential competing interests exist.

A Problem, Solution, Validation Impact Canvas is a simplified visual ideation mapping canvas allowing users to identify the core Problem, Solutions and Validation actions associated their target problem area.

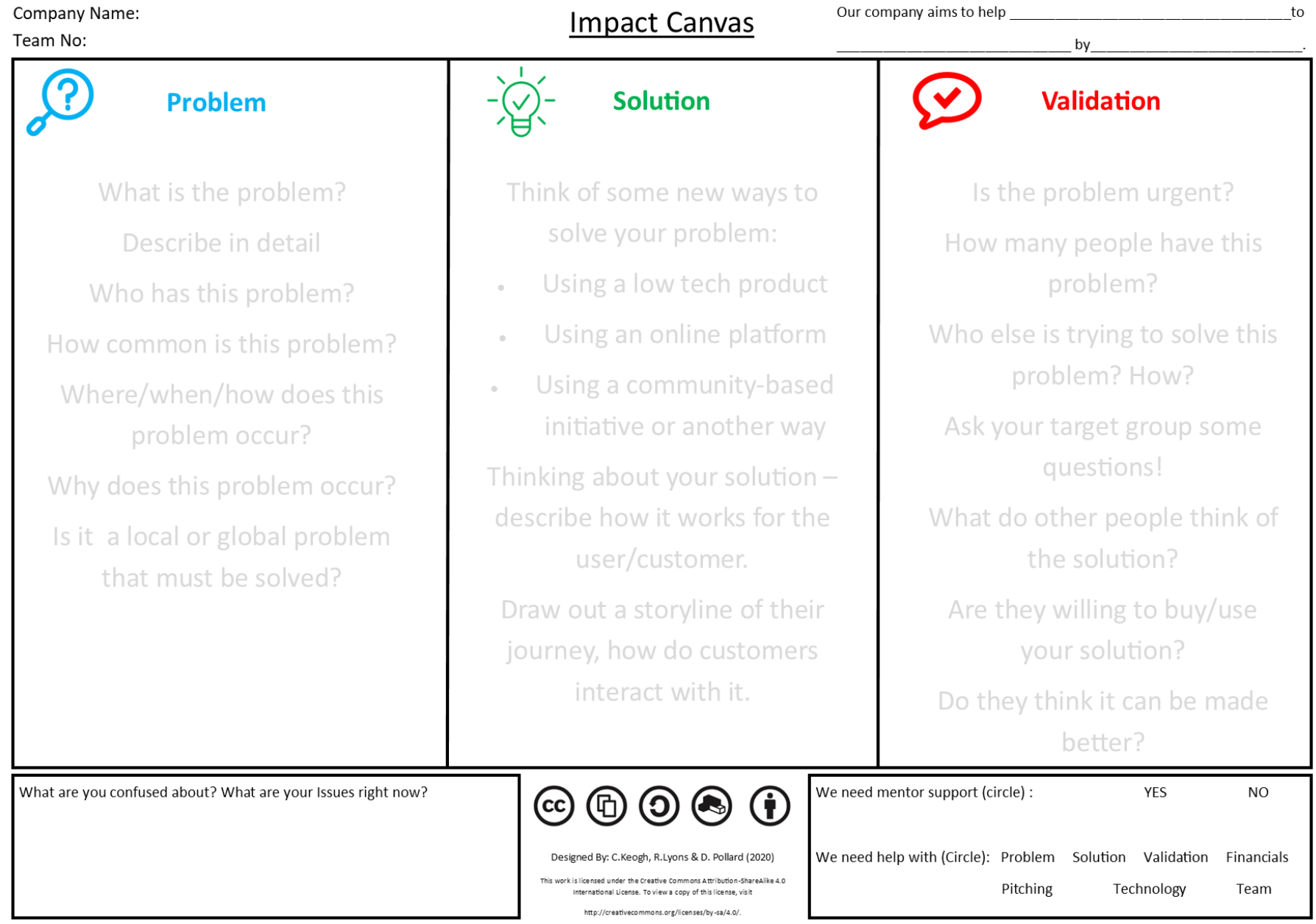

